\title{
BMJ Open Enhancing recognition of obstetric anal sphincter injuries in six maternity units in Palestine: an interventional quality improvement study
}

\author{
Hadil Ali-Masri, ${ }^{1,2,3}$ Sahar Hassan, ${ }^{4,5}$ Khaled Ismail, ${ }^{6}$ Kaled Zimmo, ${ }^{1,2,7}$ \\ Mohammed Zimmo, ${ }^{1,2,8}$ Erik Fosse, ${ }^{1,2}$ Åse Vikanes, ${ }^{1}$ Katariina Laine ${ }^{9,10}$
}

To cite: Ali-Masri $\mathrm{H}$, Hassan $\mathrm{S}$ Ismail $\mathrm{K}$, et al. Enhancing recognition of obstetric anal sphincter injuries in six maternity units in Palestine: an interventional quality improvement study. BMJ Open 2018;8:e020983. doi:10.1136/ bmjopen-2017-020983

\section{- Prepublication history for} this paper is available online. To view these files, please visit the journal online (http://dx.doi. org/10.1136/bmjopen-2017020983).

Received 4 December 2017 Revised 15 May 2018 Accepted 24 May 2018

Check for updates

For numbered affiliations see end of article.

Correspondence to

Dr Hadil Ali-Masri;

had_yousif@yahoo.ca

\section{ABSTRACT}

Objective To explore the impact of a training intervention on obstetric anal sphincter injuries' (OASIS) detection rate.

Design Prospective quality improvement interventional study.

Setting Six secondary and tertiary maternity units in Palestine.

Population Women having singleton vaginal births $\geq 23$ weeks' gestation or babies weighing $\geq 500 \mathrm{~g}$ ( $\mathrm{n}=22$ 922). Caesarean births $(n=5431)$, multiple gestations $(n=443)$ and vaginal births of unregistered perineum status $(n=800)$ were excluded.

Interventions Training programme for enhancing OASIS detection was conducted between 31 January and 31 December 2015. International experts delivered 2-day standardisation workshop teaching OASIS diagnosis and repair to each maternity unit. They also provided additional training to three research fellows employed in three of the maternity units. This was followed by 13 -week period of data collection (phase 1). Research fellows then delivered training intervention over 15-week interval (phase 2), including theoretical teaching and 'onsite' training in perineal trauma assessment within the six maternity units. Finally, 13-week postintervention observation (phase 3) followed.

Primary outcome measure OASIS rates were used as surrogate for OASIS recognition. OASIS rates were compared between different phases and between the two maternity unit groups (research fellow and non-research fellow based) using Pearson's $\chi^{2}$ test.

Results A total 22922 women were included. Among primiparous women, OASIS rate was higher in phase $2(2.8 \%, p<0.001)$ and phase $3(3.1 \%, p<0.001)$ than phase $1(0.5 \%)$. However, no significant differences were detected in the rates of severe OASIS (third-degree $3 \mathrm{c}$ and fourth-degree tears) between phase 1 and $2(0.5 \%$ vs $0.3 \%$ ), because this would have required at least 103 women with severe OASIS to be included in each phase. Among parous women, OASIS rate was significantly higher in phase $2(0.6 \%, p=0.002)$ but not in phase 3 $(0.4 \%, p=0.071)$ compared with phase $1(0.2 \%)$. Research fellows' maternity units showed higher OASIS rates among primiparous women in phase 2 ( $3.6 \%$ vs $1.4 \%, p=0.001$ ) and phase $3(4.3 \%$ vs $0.8 \%, p<0.001)$ than non-research fellows' maternity units.
Strengths and limitations of this study

- The study highlights a critical birth complication which is neglected in many settings especially in low-income countries.

- The geographical distribution of the participating maternity units and the large study population are representative and of sufficient size to generalise the study findings.

- One-to-one training approach enabled better understanding of the trainees' needs, improved communication and provided more opportunities to practice new skills under supervision.

- The expert workshop might have contaminated the reference data which was collected before the local training intervention.

- Interruption of the training intervention in one of the non-research fellows' maternity units may have overestimated the difference between research fellows' and non-research fellows' maternity units.

Conclusions This work is basically an epidemiological study which has identified the prevalence of perineal lacerations and their severity on a large sample of women representative of an entire geographical ethnic region. The quality improvement intervention improved OASIS detection mainly in the research fellows' maternity units. Regular mandatory national programmes in obstetric perineal trauma assessment and management by local champions are essential to mitigate the risk of missing significant degrees of trauma.

\section{INTRODUCTION}

Obstetric anal sphincter injuries (OASIS) are severe perineal tears involving disruption of one or both anal sphincter muscles with or without breach of the rectal mucosa. ${ }^{1}$ In addition to sexual dysfunction and perineal pain, ${ }^{2}$ up to $50 \%$ of women with history of OASIS develop some form of anal incontinence. ${ }^{3-5}$ Association with urinary incontinence has also been described. ${ }^{34}$ 
Many countries have observed an overall increase in OASIS rate during the last decade ${ }^{6}{ }^{7}$ Differences in OASIS rates have been reported to range from $1 \%$ to $6 \% .{ }^{8-13}$ Improved diagnostic skills and recording, but also discrepancies in clinical practices explain OASIS rates' variations over time, between countries and maternity units. $^{8-13}$

Proper instant repair of OASIS reduces the risk of developing anal incontinence. ${ }^{1415}$ Therefore, doctors and midwives should be able to classify perineal tears accurately. OASIS recognition, however, may be challenging for practitioners. Sultan and Fernando ${ }^{16}$ have previously reported that more than half of the practising obstetricians in the UK were unsatisfied with their training regarding perineal trauma diagnosis and repair.

In Palestine, perineal trauma management lacked systematic and evidence-based clinical practice. Furthermore, OASIS rate in Palestine has been unknown until this work was undertaken and therefore has been of little concern among obstetricians, midwives and policy-makers assuming that this event was rare. In view of that, local interventional training programme was developed to shed light on and improve OASIS recognition in Palestine. The main aims of this study were to explore OASIS rate and to assess the trends of OASIS rate in six Palestinian maternity units prior to, during and following a quality improvement intervention.

\section{METHODS}

A prospective interventional quality improvement study was conducted in six maternity units in Palestine between 31 January 2015 and 31 December 2015. The participating maternity units were selected based on their capacity and the geographical distribution of their locations. Three maternity units were located in Gaza and three in the
West Bank with total of 35000-40000 births per annum. Five of the six maternity units were teaching centres providing training programmes for medical students, interns, resident doctors and student midwives. One of the research fellows involved in this study works at the non-teaching maternity unit. The classification systems and repair techniques of perineal tears did not follow a consolidated approach based on updated evidence but varied widely between the six maternity units and among birth attendants within the same unit. The study design, undertaking and reporting followed the Standards for Quality Improvement Reporting Excellence 2.0 statement for quality improvement studies.

The primary outcome measure was OASIS rate which was used as surrogate for OASIS recognition rate and calculated as the per cent number of OASIS cases per number of vaginal births. The study population included all women who gave singleton spontaneous or operative vaginal birth at $\geq 23$ gestational weeks or $\geq 500 \mathrm{~g}$ in birth weight. Figure 1 describes the inclusion and exclusion criteria of the study population.

An expert workshop preceded the active component of the study which comprised three phases;: preclinical training observation (phase 1), training intervention (phase 2) and postintervention observation (phase 3). Figure 2 presents sequence and timeline of the study phases.

\section{Standardisation expert workshop}

Prior to commencing the study, a 2-day expert workshop was held in each of the six maternity units. The primary goal of the expert workshop was to deliver standardised evidence-based and up-to-date new approach to diagnosis and treatment of OASIS and episiotomy repair by international experts and to train three local research fellows (authors: HA-M, KZ and MZ) working in three of the

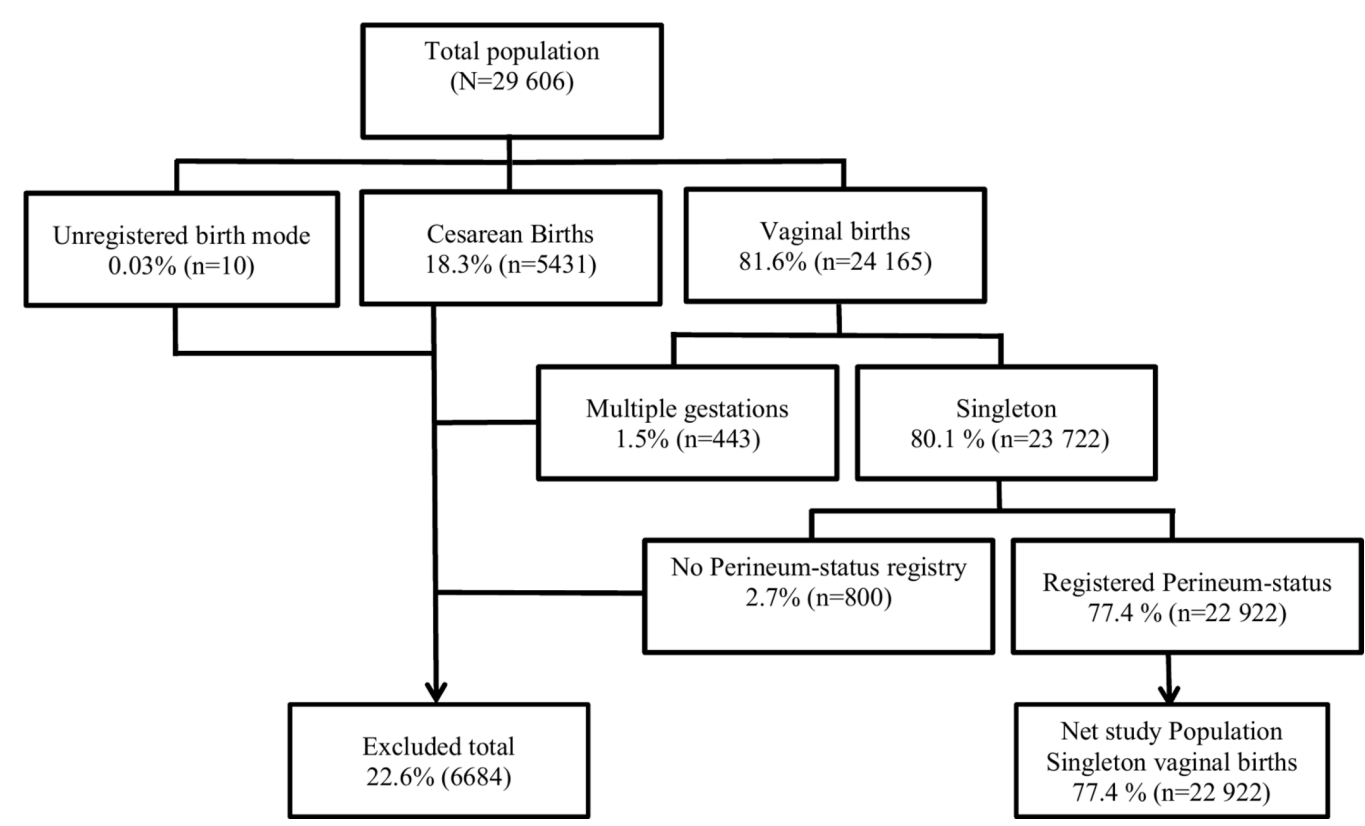

Figure 1 Flow chart describing inclusion and exclusion criteria of the study population. 


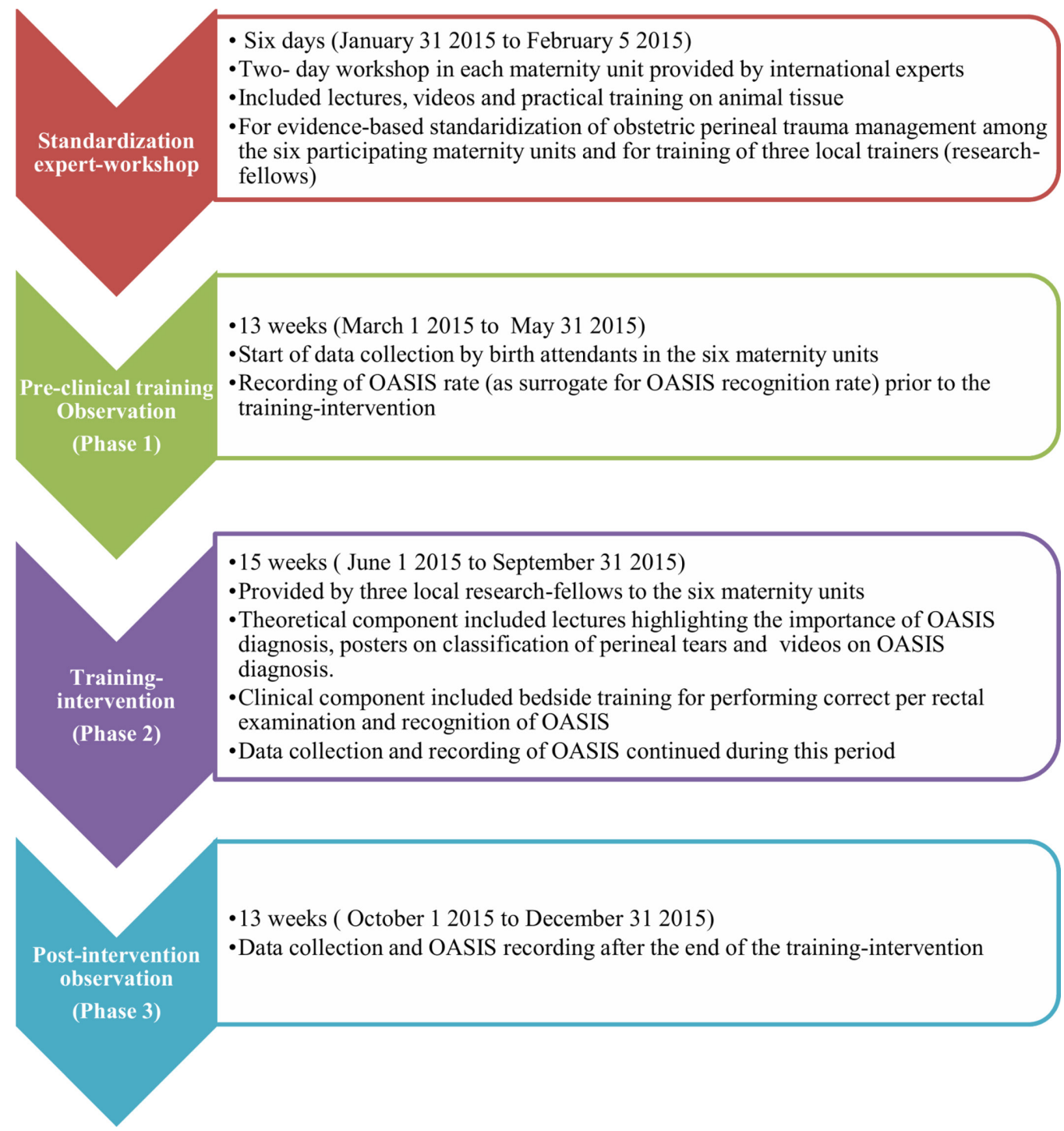

Figure 2 Flow chart demonstrating sequence and timeline of the study phases. OASIS, obstetric anal sphincter injuries.

maternity units to coordinate delivery of face-to-face training within the six maternity units. A total of $86(86 \%)$ midwives and $116(90.6 \%)$ doctors received lectures, video sessions and practical training on animal models (as illustrated by Zimmo et $a l^{17}$ ) to standardise perineal trauma assessment and repair technique before collecting any information. The training was based on the Royal College of Obstetricians and Gynecologists (RCOG) and National Institute for Health and Care Excellence (NICE) guidelines. ${ }^{18} 19$ Additionally, each maternity unit was donated a training package in obstetric perineal trauma including a book, DVD and perineal repair simulator (www.perineum. net) which were stored until the research fellows used them for further training.

\section{Preclinical training observation (phase 1)}

The expert workshop was shortly followed by 13 weeks' observational period to collect data and record OASIS rate in the six maternity units prior to the local training intervention.

\section{Training intervention (phase 2)}

The quality improvement training intervention was conducted by the three local research fellows (consultants of obstetrics and gynaecology). The goals of this intervention were to improve birth attendants' skills in OASIS diagnosis and to nominate champions who would train those who were not directly trained by the research fellows during this period. The intervention was delivered to the West Bank and Gaza concurrently. One research fellow (HA-M) undertook the intervention in the three West Bank maternity units and two research fellows ( $\mathrm{KZ}$ and $\mathrm{MZ}$ ) in the three Gaza maternity units. Resources used for the intervention were the RCOG and NICE guidelines, ${ }^{18}{ }^{19}$ and the training package provided by the international experts. The three research fellows trained $66(80.5 \%)$ doctors and $50(79.4 \%)$ midwives in the three units where they work (research fellows' maternity units) and 35 (76.1\%) doctors and $30(81.1 \%)$ midwives in the other three units. Those who were not trained were on maternity 
leave or whose working schedule did not overlap with that of the research fellows.

The intervention entailed a 15-week multitask educational programme including the following interactive theoretical and clinical components:

- Posters on perineal tears classification.

- Lectures during morning meetings, on OASIS rates and complications, designed to help change practitioners' perception about the 'stigma' of OASIS detection and emphasise the importance of OASIS recognition.

- Perineal anatomy and OASIS assessment video demonstrations to familiarise birth attendants with the condition before receiving active bedside training. The videos were displayed to groups during morning meetings and to individuals during their working shifts.

- Reminder wall signs fitted above delivery beds to remind birth attendants of rectal examination every time they attend a birth.

- One-to-one bedside clinical training by the research fellows who were available in labour rooms 5 to 8 hours a day during morning or evening shifts for 4 weeks per maternity unit. For each maternity unit, 2 of the 4 weeks of the bedside training were separate and two were consecutive and distributed throughout phase 2. This timeline was planned to overlap with the schedule for as many trainees as possible.

The supervised training included identification of perineal anatomy under real conditions, performing correct rectal examination and vaginal exploration for women immediately after giving birth. In order to confirm the accuracy of the diagnosis, the research fellows reassessed women examined by the trainee birth attendant.

The plan was to deliver the same training intervention to the six maternity units. However, due to unforeseen circumstances, this training was not achieved to the same level in one of the non-research fellows' maternity units.

\section{Postintervention observation (phase 3)}

The postintervention observation traced OASIS rate for 13 weeks in the six maternity units following the training intervention.

\section{Data collection}

Data were collected prospectively, during phases 1,2 and 3 , by birth attendants (doctors and midwives) after they received training in data registration and entry. The procedure for data management and assessment of its completeness and reliability was previously described by Hassan et al..$^{20} \mathrm{~A}$ case registration form was filled for each woman admitted to labour room during the study period. Data were entered into web-based health information system (dhis2, version 2.20) developed by Global infrastructure department at University of Oslo. Dhis2 is currently used in more than 47 low/middle-income countries as national health information system for data registration and management and health programme monitoring. Data were transferred from dhis2 to be stored in Service for Sensitive Data (TSD) platform. TSD facilities, owned by the University of Oslo, is developed and operated by TSD service group at the University of Oslo, IT Department (tsd-drift@usit.uio.no), and is used by researchers working at the university and in other public research institutions to collect, store, analyse and share sensitive data in compliance with the Norwegian regulations regarding individuals' privacy.

To assess the impact of the training intervention, OASIS rate variations were measured between the three study phases. This variation was considered a surrogate for the impact of the quality improvement intervention on increasing awareness and assessment skills and hence trauma recognition.

\section{Statistical analysis}

SPSS program (V.22.0) was used for statistical analysis. Descriptive analysis were used to present frequencies, means and SDs. Continuous variables were checked for normality using Shapiro-Wilk test. The three study phases' clinical characteristics were compared using Pearson's $\chi^{2}$ test, for categorical variables, and one-way analysis of variance test, for continuous variables. Variations in OASIS rate between the three study phases were compared using Pearson's $\chi^{2}$ test or Fisher's exact test (for cells with counts $<5$ ). Logistic regression was applied to assess whether the observed outcomes were due to the training intervention or some other causes (confounders such as episiotomy and primiparity). OASIS rate was explored in subgroups according to gestational age and mode of delivery. A subanalysis by type of maternity unit was also done. Maternity units were classified, according to whether one of the research fellows was based in them or not, into research fellows' and non-research fellows' maternity units and were compared using Pearson's $\chi^{2}$ test for categorical variables and independent t-test for continuous variables. The same analysis was performed excluding the unit with interrupted training intervention. To estimate the direct impact of maternity unit type on OASIS rate, logistic regression analysis was performed to adjust for confounders (episiotomy, operative birth and birth weight). Level of significance was set at $p$ value $<0.05$.

\section{Ethical considerations}

Oslo University Hospital signed an agreement with the Palestinian Ministry of Health who approved conducting the study within its facilities. Verbal consent was obtained from all women who were examined during the training, immediately before the repair, for rectal examination and illustration to the attending doctor or midwife. Women could not choose to be operated by a tutor or trainee but, if OASIS is suspected, they should be re-examined and sutured by an experienced obstetrician.

\section{Patient and public involvement}

Neither patients nor public were involved in the design or conduct of the study. 
Table 1 Maternal and fetal characteristics in the three study phases

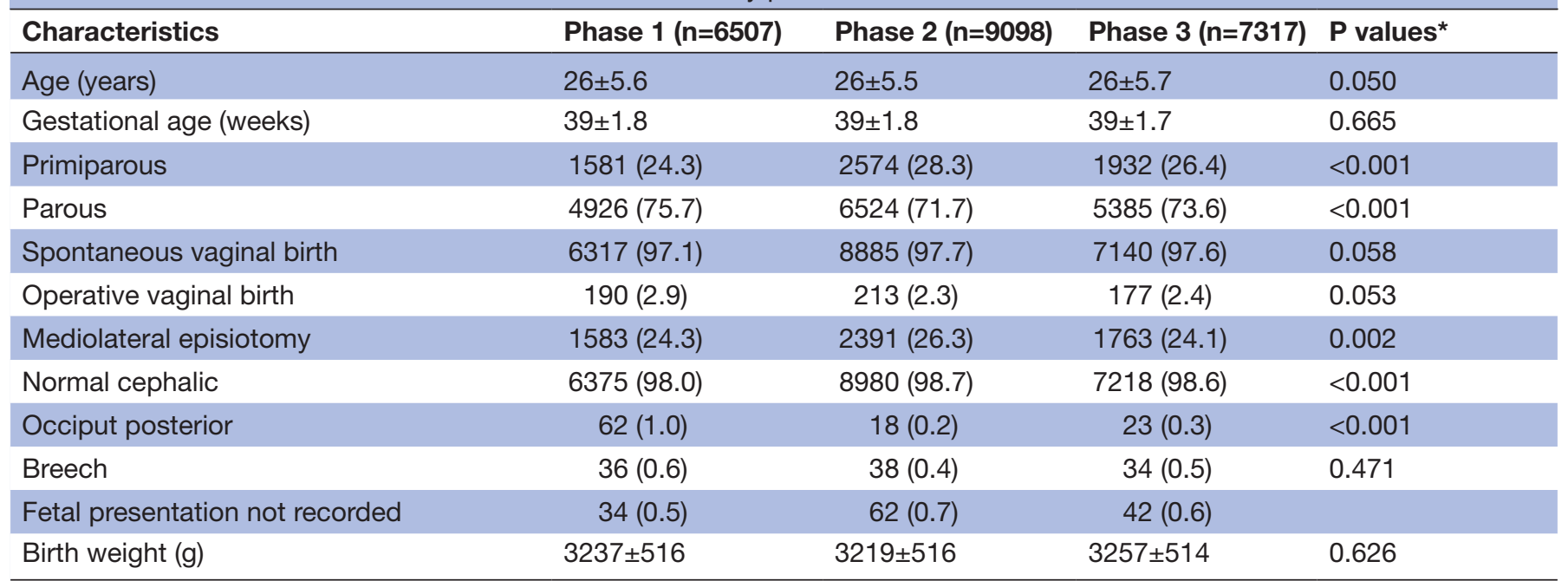

Categorical data are presented by $\mathrm{n} / \mathrm{N}(\%)$, and continuous data are presented by means \pm SDs.

*Variations were assessed using Pearson's $\chi^{2}$ test for categorical variables and one-way analysis of variance for continuous variables.

\section{RESULTS}

A net of 22922 singleton vaginal births were included. Operative vaginal birth contributed $2.5 \% \quad(n=580) ; 99.1 \%$ $(\mathrm{n}=575)$ vacuum and $0.9 \%(\mathrm{n}=5 / 580)$ forceps. Primiparous women contributed $26.6 \%(\mathrm{n}=6087)$ and parous women $73.4 \% \quad(n=16835)$ of total births. Sixty-eight per cent $(n=4142 / 6087)$ of primiparous women and $40.4 \%(\mathrm{n}=6805 / 16835)$ of parous women were delivered by obstetricians. The remaining 32\% ( $\mathrm{n}=1945 / 6087)$ of primiparous women and 59.6\% ( $\mathrm{n}=10030 / 16835)$ of parous women were delivered by midwives.

OASIS rate was explored in different gestational weeks' subgroups: $\leq 33^{+6}, 34-36^{+6}, 37-39^{+6}$ and $\geq 40$. No OASIS were identified in the first subgroup $\left(\leq 33^{+6}\right)$. OASIS rate did not vary significantly between the three other subgroups $(1.0 \% \quad(n=8 / 839)$ vs $1.0 \% \quad(n=86 / 8737)$ vs $0.9 \%(\mathrm{n}=111 / 13035), \mathrm{p}=0.272)$.

Throughout the study period, OASIS rate was significantly higher in operative birth than that in spontaneous birth (4.5\% (26/580) vs $0.8 \%(179 / 22342), \mathrm{p}<0.001)$; phase $1(1.6 \%(3 / 191)$ vs $0.2 \% \quad(14 / 6316), \mathrm{p}=0.012)$; phase $2(7.1 \%(15 / 212)$ vs $1.1 \%(94 / 8886), \mathrm{p}<0.001)$ and phase $3(4.5 \%(8 / 177)$ vs $1.0 \%(71 / 7140), p=0.001)$.

The three study phases had similar maternal and fetal characteristics except for proportions of primiparous women, mediolateral episiotomy and occiput posterior presentations (table 1 ).

Table 2 demonstrates trends of perineal status in primiparous and parous women during the three study phases using phase 1 as a reference.

Proportions of first-degree tears were significantly reduced during phase 2 and phase 3, while no statistically significant difference was found in proportions of second-degree tears between the three study phases (table 2).

In parous women, OASIS rate was significantly higher during phase 2 than phase $1(0.6 \%(n=36 / 6524)$ vs $0.2 \%$
( $n=9 / 4926), p=0.002)$. During phase 3 , there was twofold increase in OASIS rate relative to phase 1 , yet this was not statistically significant $(0.4 \% \quad(n=20 / 5385)$ vs $0.2 \%$ $(\mathrm{n}=9 / 4926), \mathrm{p}=0.071)$. Moreover, OASIS rate did not change significantly between phase 2 and phase $3(0.6 \%$ $(\mathrm{n}=36 / 6524)$ vs $0.4 \% \quad(\mathrm{n}=20 / 5385), \mathrm{p}=0.152)$. Detection rate for third-degree $3 \mathrm{a}$ was significantly higher in phase $2(0.4 \%(24 / 6524)$ vs $0.1 \%(7 / 4926), \mathrm{p}=0.030)$ but not in phase $3(0.2 \%(13 / 5385)$ vs $0.1 \%(7 / 4926)$, $\mathrm{p}=0.272)$ compared with phase 1 . No significant variation between phases 2 and 3. For higher degrees of OASIS, the detection rate was significantly higher in phase 2 $(0.2 \%(12 / 6524)$ vs $0.04 \%(2 / 4926), \mathrm{p}=0.032)$ but not in phase $3(0.1 \%(7 / 5385)$ vs $0.04 \%(2 / 4926), \mathrm{p}=0.183)$ compared with phase 1 . There was no significant difference between phases 2 and 3 .

A significant reduction in episiotomy rate was also observed together with an increased proportion of intact perineum during phase 2 and phase 3 (table 2 ).

Overall, the participating units had significantly higher OASIS rates after compared with before the training intervention (OR: 4.4, CI: 2.7 to 7.3, p<0.001). This finding remained significant (adjusted OR (aOR): 4.3, CI: 2.6 to 7.2, $\mathrm{p}<0.001$ ) after adjusting for confounders (primiparity, episiotomy, operative birth and occiput posterior).

Clinical characteristics among primiparous women in research fellows' maternity units were compared with those in non-research fellows' maternity units. Compared with non-research fellows' maternity units, research fellows' maternity units had higher episiotomy rate $(79.2 \% \quad(n=3001 / 3791)$ vs $75.1 \% \quad(n=1724 / 2296)$, $\mathrm{p}<0.001)$, higher OASIS rate $(3.1 \% \quad(\mathrm{n}=119 / 3791)$ vs $0.9 \%(\mathrm{n}=21 / 2296), \mathrm{p}<0.001)$, higher mean birth weight $(3129 \mathrm{~g}$ vs $3057 \mathrm{~g}$, mean difference $=72, \mathrm{p}<0.001)$ and lower operative birth rate $(5.6 \%(\mathrm{n}=213 / 3791)$ vs $7.4 \%$ ( $\mathrm{n}=169 / 2296), \mathrm{p}=0.007)$. 
Table 2 Trends of perineal status among primiparous and parous women in the three study phases: phase 1 was set as reference and compared with phase 2 and phase 3

\begin{tabular}{|c|c|c|c|c|c|}
\hline Perineum status & Phase 1, n/N (\%) & Phase 2, n/N (\%) & P values ${ }^{*}$ & Phase 3, n/N (\%) & $P$ valuest \\
\hline Primiparous women & $n=1581$ & $\mathrm{n}=2574$ & & $n=1932$ & \\
\hline Intact & $214(13.5)$ & $348(13.5)$ & 0.988 & $292(15.1)$ & 0.185 \\
\hline First-degree tear & $123(7.8)$ & $127(5.0)$ & 0.003 & $98(5.1)$ & 0.001 \\
\hline Second-degree tear & $42(2.7)$ & $55(2.1)$ & 0.686 & $41(2.1)$ & 0.433 \\
\hline Episiotomy without OASIS & $1194(75.5)$ & $1971(76.6)$ & 0.330 & $1442(74.6)$ & 0.455 \\
\hline OASIS with episiotomy & $5(0.3)$ & $62(2.4)$ & & $51(2.6)$ & \\
\hline OASIS without episiotomy & $3(0.2)$ & $11(0.4)$ & & $8(0.4)$ & \\
\hline OASIS total & $8(0.5)$ & $73(2.8)$ & $<0.001$ & $59(3.1)$ & $<0.001$ \\
\hline Degree 3ał & $4(0.3)$ & $61(2.4)$ & & $41(2.1)$ & \\
\hline Degree 3b§ & $2(0.1)$ & $10(0.4)$ & & $14(0.7)$ & \\
\hline Degree 3cף & $1(0.1)$ & $2(0.1)$ & & $3(0.2)$ & \\
\hline Fourth-degree tear** & $1(0.1)$ & - & & $1(0.1)$ & \\
\hline Parous women & $n=4926$ & $n=6524$ & & $n=5385$ & \\
\hline Intact & 3809 (77.3) & $5168(79.2)$ & 0.015 & $4393(81.6)$ & $<0.001$ \\
\hline First-degree tear & $652(13.2)$ & 869 (13.3) & 0.702 & $617(11.5)$ & 0.001 \\
\hline Second-degree tear & $77(1.6)$ & $98(1.5)$ & 0.669 & $94(1.7)$ & 0.596 \\
\hline Episiotomy without OASIS & $379(7.7)$ & $353(5.4)$ & $<0.001$ & $261(5.0)$ & $<0.001$ \\
\hline OASIS with episiotomy & $5(0.1)$ & $5(0.08)$ & & $9(0.17)$ & \\
\hline OASIS without episiotomy & $4(0.1)$ & $31(0.5)$ & & $11(0.2)$ & \\
\hline OASIS total & $9(0.2)$ & $36(0.6)$ & 0.002 & $20(0.4)$ & 0.071 \\
\hline Degree $3 a$ & $7(0.1)$ & $24(0.4)$ & & $13(0.2)$ & \\
\hline Degree 3b & $2(0.04)$ & $5(0.1)$ & & $6(0.1)$ & \\
\hline Degree 3c & - & $2(0.03)$ & & - & \\
\hline Fourth-degree tear & - & $5(0.1)$ & & $1(0.02)$ & \\
\hline
\end{tabular}

Differences were assessed using Pearson's $\chi^{2}$ test.

${ }^{*}$ Assesses difference between phase 1 and phase 2 .

†Assesses difference between phase 1 and phase 3 .

łLess than $50 \%$ of external anal sphincter is torn.

§More than $50 \%$ of external sphincter is torn.

ๆExternal and internal sphincters are torn.

** The anal sphincter muscles and the rectal mucosa are torn.

OASIS, obstetric anal sphincter injuries.

Figure 3 demonstrates trends of OASIS rate among primiparous women in research fellows' maternity units and non-research fellows' maternity units through the three study phases. In research fellows' maternity units, OASIS rate was significantly higher during phase $2(3.6 \%$ $(\mathrm{n}=61 / 1698)$ vs $0.5 \%(\mathrm{n}=4 / 823), \mathrm{p}<0.001)$ and phase 3 $(4.3 \%(\mathrm{n}=54 / 1270)$ vs $0.5 \%(\mathrm{n}=4 / 823), \mathrm{p}<0.001)$ than phase 1 . However, there was no significant difference between phase 2 and phase $3(3.6 \% \quad(n=61 / 1698)$ vs $4.3 \%$ $(\mathrm{n}=54 / 1270), \mathrm{p}=0.357)$. In non-research fellows' maternity units, there was a statistically insignificant increase in OASIS rate during phase 2 compared with phase 1 (1.4\% $(\mathrm{n}=12 / 876)$ vs $0.5 \% \quad(\mathrm{n}=4 / 758), \mathrm{p}=0.129)$ followed by reduction to $0.8 \%(\mathrm{n}=5 / 662)$ during phase 3 .

Among primiparous women, OASIS rate in research fellows' maternity units was compared with that in non-research fellows' maternity units during each of the three study phases. Both groups had recorded OASIS rate of $0.5 \%$ during phase 1. Ensuing the training intervention, OASIS rate was significantly and persistently higher in research fellows' maternity units than that in non-research fellows' maternity units; phase 2 (3.6\% $(\mathrm{n}=61 / 1698)$ vs $1.4 \%(\mathrm{n}=12 / 876), \mathrm{p}=0.001)$ and phase $3(4.3 \%(\mathrm{n}=54 / 1270)$ vs $0.8 \%(\mathrm{n}=5 / 662), \mathrm{p}<0.001)$. This difference remained significant even after excluding the non-research fellows' maternity unit where one-to-one training was suboptimal (table 3 ).

Using logistic regression analysis, OASIS rate was significantly dependent on the type of maternity unit before and after adjusting for episiotomy, operative birth and birth weight (research fellows' vs non-research fellows' maternity units; aOR: 2.0, CI: 1.1 to $3.5, \mathrm{p}<0.029$ ).

Among parous women, OASIS rate in research fellows' maternity units increased significantly in phase $2(0.7 \%$ 
$\rightarrow$ Research fellows'-maternity units $\rightarrow$-Non-research fellows'-maternity units

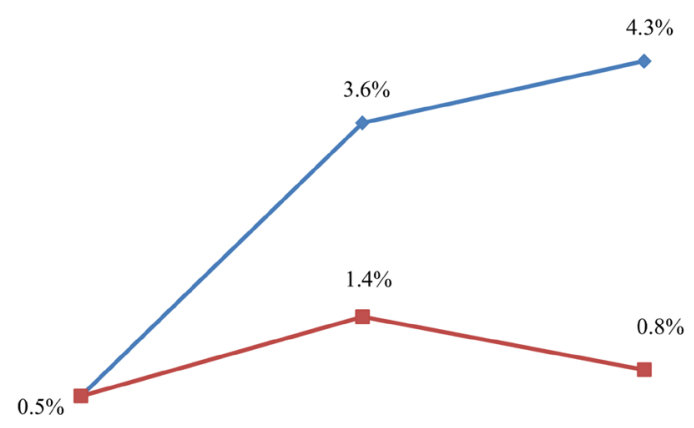

Phase 1

Phase 2

Phase 3

Figure 3 Graph demonstrating trends of obstetric anal sphincter injuries (OASIS) rate among primiparous women in research fellows' maternity units and non-research fellows' maternity units during the three study phases.

$(\mathrm{n}=29 / 3932)$ vs $0.2 \%(\mathrm{n}=6 / 2739), \mathrm{p}=0.004)$ and insignificantly in phase $3(0.5 \%(n=15 / 3034)$ vs $0.2 \%(n=6 / 2739)$, $\mathrm{p}=0.083$ ) compared with phase 1 . Additionally, no significant difference was detected between phase 2 and phase $3(0.7 \% \quad(n=29 / 3932)$ vs $0.5 \% \quad(n=15 / 3034), p=0.204)$. In the non-research fellows' maternity units, OASIS rate among parous women showed no significant differences between phase 2 and phase $1(0.3 \%(n=7 / 2592)$ vs $0.1 \%(\mathrm{n}=3 / 2187), \mathrm{p}=0.361)$, phase 3 and phase 1 $(0.2 \%(\mathrm{n}=5 / 2351)$ vs $0.1 \%(3 / 2187), \mathrm{p}=0.728)$ or phase 2 and phase $3(0.3 \%(n=7 / 2592)$ vs $0.2 \%(n=5 / 2351)$, $\mathrm{p}=0.682)$.

OASIS rates were higher in births attended by doctors than those attended by midwives during the three study phases: phase $1(0.4 \%(n=11 / 2633)$ vs $0.2 \%(n=6 / 3874)$, $\mathrm{p}=0.049)$, phase $2 \quad(1.7 \% \quad(\mathrm{n}=77 / 4441) \quad$ vs $\quad 0.7 \%$ $(\mathrm{n}=32 / 4657), \mathrm{p}<0.001)$ and phase $3(1.4 \%(\mathrm{n}=56 / 3873)$ vs $0.7 \%(\mathrm{n}=23 / 3444), \mathrm{p}=0.001)$.

Table 3 OASIS rates during the three study phases in the research fellows' maternity units and non-research fellows' maternity units excluding the maternity unit where the training intervention was suboptimal

$\begin{array}{lllll}\begin{array}{l}\text { OASIS } \\ \text { rate } \\ \%(n / N)\end{array} & \begin{array}{l}\text { Study } \\ \text { phase }\end{array} & \begin{array}{l}\text { Research } \\ \text { fellows' } \\ \text { maternity } \\ \text { units }\end{array} & \begin{array}{l}\text { Non- } \\ \text { research } \\ \text { fellows' } \\ \text { maternity } \\ \text { units }\end{array} & \text { P value* }^{*} \\ & \text { Phase 1 } & 0.5(4 / 823) & 0.4(2 / 534) & >0.999 \\ & \text { Phase 2 } & 3.6(61 / 1698) & 1.4(10 / 710) & 0.004 \\ & \text { Phase 3 } & 4.3(54 / 1270) & 0.7(4 / 535) & <0.001\end{array}$

*Differences assessed by Pearson's $\chi^{2}$ test or Fisher's exact test for cells with counts $<5$.

$\mathrm{n}$, number of OASIS; $\mathrm{N}$, total vaginal births per phase in either research fellows' or non-research fellows' maternity units; OASIS, obstetric anal sphincter injuries.

\section{DISCUSSION}

An overall increase in OASIS rate has been observed after the quality improvement intervention (primiparous women $0.5 \%$ to $3.1 \%$ and parous women $0.2 \%$ to $0.4 \%$ ). The change in the OASIS recognition rate was recorded after face-to-face clinical training following a 2-day expert workshop aimed at improving the diagnostic and therapeutic skills of the medical and obstetric staff. The training intervention, in this study, did not involve OASIS prevention strategies nor interfered with the routine intrapartum management protocols. Women often give birth in lithotomy or supine positions. Epidural analgesia was not available in the study maternity units, but opioids might have been given during the first stage of labour. Traditional manual perineal support and mediolateral episiotomy were routinely used before and during the three study phases. Other perineal protection measures such as warm compresses and perineal massage are not used in the participating maternity units.

Given that all intrapartum management protocols remained unchanged throughout the study period and that all possible confounders have been adjusted for, the only plausible explanation for the increased OASIS rate is improved OASIS recognition as a result of the training intervention. The improvement level was better in the research fellows' maternity units whose staff were more receptive to suggested changes. Accordingly, training delivered by clinicians within their working places seemed to amplify and sustain the pursued outcome of the intervention. This conclusion concurs with other studies' findings where the presence of local champions has been associated with success of the quality improvement intervention. ${ }^{21}$

The maternity units' geographical distribution and the large study population are representative and of sufficient size to generalise the study findings. This work is basically an epidemiological study which has identified the prevalence of perineal lacerations and their severity on a large sample of women representative of an entire geographical ethnic region. One-to-one training approach enabled better understanding of the trainees' needs, improved communication and provided more opportunities to practice new skills under supervision. The training intervention, however, faced few challenges such as:

- Rectal examination for assessing the degree of trauma was not routinely performed before this study and therefore, some birth attendants showed resistance to taking the rectal examination as a routine clinical practice.

- Busy clinical setting and work overload which caused interruption and inadequate training of some birth attendants.

- Few birth attendants avoided the clinical training due to lack of interest, dissatisfaction to be trained by individuals from a different maternity unit, and more importantly due to their perceived stigma associated with OASIS complicating a birth which made them 
feel uneasy about their practice being observed and judged by the research fellows.

- One-to-one clinical training was effortful and time-consuming as it was influenced by birth attendants' working schedule and the availability of vaginal births during the working hours of the research fellows.

Despite these challenges, the training intervention seemed to have an overall positive impact. This suggests that this intervention was still effective in less ideal and more real-life situations. Moreover, although only speculative, we believe that training by local champions within their working places is more sustainable and cost-effective compared with workshops delivered by international experts. Hence, we believe that this quality improvement intervention can be applicable to other low/middle-income health care settings.

Different OASIS detection rates before and after the training intervention indicates that large portion of those injuries was left undiagnosed and consequently not well-repaired. Up to $2.6 \%(n=159)$ of OASIS were potentially missed among primiparous women, as indicated by the difference in OASIS rate between phase $1(0.5 \%)$ and phase $3(3.1 \%)$. If any of these injuries are identified in the future, they would have been considered occult OASIS when in fact they have been missed. In a cohort study of primiparous women who underwent endoanal ultrasound 5 months postpartum to examine their external sphincter muscles, 28\% women had sphincter injuries of which $87 \%$ were not identified at birth. ${ }^{22}$ Another study showed that the prevalence of third-degree tears increased from $11 \%$ to $25 \%$ in 241 primiparous women when they were re-examined by an experienced research fellow. ${ }^{23}$ Missed OASIS results in serious implications on women's quality of life and the risk of anal incontinence is increased after successive deliveries. ${ }^{24} 25$ These complications are, arguably, higher for Palestinian women who tend to have many children (fertility rate in Palestine 4.1 births per woman). ${ }^{26}$

Birth attendants in this study lacked a proper training in perineal trauma assessment resulting in inadequate OASIS detection. The training intervention was the first specialised training programme on obstetric perineal trauma in Palestine. Training insufficiency has also been described in high-income countries. In the UK, a survey including 150 doctors and midwives indicated that less than $20 \%$ doctors and $50 \%$ midwives were satisfied with their training in OASIS diagnosis and repair. ${ }^{27}$ In contrast, no studies assessed the availability or quality of such training programmes in other low-income countries. A cross-sectional study in 24 low-income countries reported that OASIS prevalence varied between $0.1 \%$ and $15 \%$, which was assumed to be caused by either underdiagnosis or overdiagnosis, highlighting the need for more training in low-income countries. ${ }^{28}$

Despite the expert workshop, episiotomy continued to be routinely used in primiparous women $(75.8 \%$ to $79 \%)$. This is not surprising, as changing the clinical practice is often faced with challenges resulting in delayed implementation of the new evidence-based guidelines. ${ }^{29}$

After the training intervention, we have observed that OASIS in primiparous women was more frequently associated with delivery with episiotomy (phase $2 ; 2.4 \%$ vs $0.4 \%$ and phase $3 ; 2.6 \%$ vs $0.4 \%$ ) than delivery without episiotomy. However, this data should be interpreted with caution because of the small number of women in this group who delivered without an episiotomy and because of the nature of the study design.

It could be argued that OASIS rate was higher in research fellows' maternity units where episiotomy use was higher. However, we have showed earlier, in this study, that the type of maternity unit was independently associated with OASIS rate after adjustment for episiotomy and other potential confounders. It is also possible that the observed difference in OASIS rates between research fellows' and non-research fellows' maternity units is a reflection of the difference in the adoption of and adherence to the training.

The improved detection rate of OASIS among primiparous women was significant for third-degree 3a during phases 2 and 3 . While for more severe degrees of OASIS (degree 3b, 3c and fourth-degree tears), there was a trend of better detection rate which was statistically significant during phase 3 but not in phase 2 compared with phase 1 . This could be due to the smaller number of women who sustained such severe degrees of trauma or because of a higher chance of missing an OASIS prior to training if it was $3 \mathrm{a}$ compared with $3 \mathrm{~b}, 3 \mathrm{c}$ and fourth-degree tears. In order to be able to detect significant differences in the rates of severe OASIS between phase 1 and $2(0.5 \%$ vs $0.3 \%$ ), at least 103 women with severe OASIS are needed to be included in each phase.

In addition to the increased OASIS rate, primiparous women have shown an increase in the number of tears classified as second degree and a concomitant reduction in tears classified as first degree. Earlier studies have shown that clinicians tend to underestimate perineal trauma and therefore classify third-degree tears as second-degree tears. ${ }^{27} 30$

Our findings suggest that the training intervention has improved the accuracy of the diagnosis by introducing a standardised classification system, confirming the importance of rectal examination and providing the one-to-one training which allowed the tutors to immediately reassess the diagnosis made by the trainees. In contrast, the expert workshop has resulted in an improvement in the knowledge of perineal anatomy but not in the knowledge of OASIS classification. ${ }^{17}$ Nevertheless, this finding was not based on direct assessment of the clinical skills but on self-reported questionnaires, which were completed during working hours.

Among parous women, the detection rate of all degrees of OASIS increased significantly only when the research fellows were available on site. This small difference in the recognition rate, which did not reach statistical significance in the non-research fellows' maternity 
units, may be contributed to the expert workshop effect. Another speculative assumption is that midwives, who delivered the majority of parous women, have less knowledge in perineal anatomy and therefore tend to miss OASIS more than doctors. ${ }^{31}$ Zimmo et al ${ }^{17}$ described that after the expert workshop $54 \%$ midwives reported good knowledge of perineal anatomy compared with $79 \%$ doctors. This may also justify the higher OASIS rates observed in births attended by doctors.

The bedside clinical training does not seem to result in a significant increase in all degrees of OASIS recognition rate between phase 2 and 3 . This may be due to the skill gap created by staff turnover. Nonetheless, this insignificant difference may be considered a reflection of the sustained effect of the training intervention.

\section{Limitations}

It could be argued that the expert workshop might have contaminated the reference data. However, given the lack of standardised protocol for perineal trauma management, it was essential that birth attendants be given an overview of the evidence-based practice for the first time to help them understand what to look for before data collection. The differences, between phase 1 and 2, in the recognition rate could have been even greater without 2 days of the standardisation training. However, the expert workshop's practical session on animal models was quite different from the real conditions available during bedside training. In support of this view is Zimmo et al, ${ }^{17}$ finding that the 2-day expert workshop only improved theoretical knowledge as their results were based on self-reported questionnaires rather than the assessment of the actual clinical practice.

Obtaining baseline data retrospectively would have been more ideal. Unfortunately such data were not accessible in our setting where a reliable birth registry does not exist. The data on maternal and newborn's health are available as annual reports which are incomprehensive and too deficient to be used in research. ${ }^{20}$

Interruption of the training intervention in one of the non-research fellows' maternity units may have overestimated the difference between research fellows' and non-research fellows' maternity units. Yet, similar results were produced when this particular maternity unit was excluded from the analysis. Differences in OASIS rates between research fellows' and non- research fellows' maternity units were presented among primiparous women since OASIS rate among parous women was low and maybe less representative.

\section{CONCLUSION}

The quality improvement intervention has improved OASIS recognition by feasible means which can be replicated in different settings. However, we believe that many of these injuries are still missed. Therefore, integration of clinical training for perineal trauma assessment as mandatory component of midwifery and doctor residency training programmes, in addition to adherence to updated standardised protocols are recommended to sustain the impact of the intervention and to fill knowledge and skill gaps in clinical practice.

\section{Author affiliations}

${ }^{1}$ The Intervention Centre, Oslo University Hospital, Rikshospitalet, Oslo, Norway ${ }^{2}$ Institute of Clinical Medicine, Faculty of Medicine, University of Oslo, Oslo, Norway ${ }^{3}$ Department of Obstetrics, Palestine Medical Complex, Ramallah, Palestine ${ }^{4}$ Faculty of Pharmacy, Nursing and Health Professions, Birzeit University, Ramallah, Palestine

${ }^{5}$ Institute of Community and Public Health, Birzeit University, Ramallah, Palestine ${ }^{6}$ Institute of Metabolism and Systems Research, College of Medical and Dental Sciences, University of Birmingham, Birmingham, UK

${ }^{7}$ Department of Obstetrics, Al Aqsa Martyrs Hospital, Gaza, Palestine

${ }^{8}$ Department of Obstetrics, Al Shifa Hospital, Gaza, Palestine

${ }^{9}$ Department of Obstetrics, Oslo University Hospital, Ullevål, Oslo, Norway

${ }^{10}$ Department of Health Management and Health Economics, Institute for Health and Society, University of Oslo, Oslo, Norway

Acknowledgements We acknowledge Abdul Sultan, Ranee Thakar and Anny Spydslaug for their contribution in the expert workshop. We also thank doctors and midwives for their effort and time they spent in data collection.

Contributors All authors have read and approved the final version of the manuscript. HA-M: wrote the manuscript and contributed to study design, training during phase 2, data collection and data analysis. SH: contributed to study design, training planning, data collection and critical comments on manuscript. Kl: contributed to study design, training in the expert workshop and critical comments on manuscript for intellectual content. KZ: contributed to the training during phase 2 , data collection and manuscript revision. MZ: contributed to the training during phase 2, data collection and manuscript revision. EF: contributed to study design, planning and manuscript revision. ÅV: contributed to study design, the expert workshop and critical comments on the manuscript. KL: contributed to study design, planning for the training intervention, data analysis and critical comments on manuscript for intellectual content. HA-M had full access to all the data in the study and had final responsibility for the decision to submit for publication.

Funding This study was funded by the Norwegian Research Council (grant no: 234452). The fund was granted after reviewing the study proposal through a competitive procedure. The funding source had no role in the study design, data collection, data analysis, data interpretation or writing of the manuscript.

Competing interests None declared.

Patient consent Not required.

Ethics approval This study was reviewed and approved as health quality research by the Regional Committee for Medical and Health Research Ethics in South-Eastern Norway (REK 2014/1727) and the Norwegian Data Inspectorate (17/00082-2/GRA).

Provenance and peer review Not commissioned; externally peer reviewed. Data sharing statement No additional data are available.

Open access This is an open access article distributed in accordance with the Creative Commons Attribution Non Commercial (CC BY-NC 4.0) license, which permits others to distribute, remix, adapt, build upon this work non-commercially, and license their derivative works on different terms, provided the original work is properly cited and the use is non-commercial. See: http://creativecommons.org/ licenses/by-nc/4.0/

(c) Article author(s) (or their employer(s) unless otherwise stated in the text of the article) 2018. All rights reserved. No commercial use is permitted unless otherwise expressly granted.

\section{REFERENCES}

1. Harvey MA, Pierce M, Alter JE, et al. Obstetrical anal sphincter injuries (OASIS): prevention, recognition, and repair. J Obstet Gynaecol Can 2015;37:1131-48.

2. Sundquist JC. Long-term outcome after obstetric injury: a retrospective study. Acta Obstet Gynecol Scand 2012;91:715-8. 
3. Laine K, Skjeldestad FE, Sanda B, et al. Prevalence and risk factors for anal incontinence after obstetric anal sphincter rupture. Acta Obstet Gynecol Scand 2011;90:319-24.

4. Laine K, Skjeldestad FE, Sandvik L, et al. Prevalence and risk indicators for anal incontinence among pregnant women. ISRN Obstet Gynecol 2013;2013:1-8.

5. Soerensen MM, Buntzen S, Bek KM, et al. Complete obstetric anal sphincter tear and risk of long-term fecal incontinence: a cohort study. Dis Colon Rectum 2013;56:992-1001.

6. Ampt AJ, Ford JB, Roberts CL, et al. Trends in obstetric anal sphincter injuries and associated risk factors for vaginal singleton term births in New South Wales 2001-2009. Aust N Z J Obstet Gynaecol 2013;53:9-16.

7. Gurol-Urganci I, Cromwell DA, Edozien LC, et al. Third- and fourth-degree perineal tears among primiparous women in England between 2000 and 2012: time trends and risk factors. BJOG 2013;120:1516-25.

8. Laine K, Gissler M, Pirhonen J. Changing incidence of anal sphincter tears in four Nordic countries through the last decades. Eur J Obstet Gynecol Reprod Biol 2009;146:71-5.

9. Laine K, Rotvold W, Staff AC. Are obstetric anal sphincter ruptures preventable? Large and consistent rupture rate variations between the Nordic countries and between delivery units in Norway. Acta Obstet Gynecol Scand 2013;92:94-100.

10. Pirhonen JP, Grenman SE, Haadem K, et al. Frequency of anal sphincter rupture at delivery in Sweden and Finland--result of difference in manual help to the baby's head. Acta Obstet Gynecol Scand 1998;77:974-7.

11. Prager M, Andersson KL, Stephansson O, et al. The incidence of obstetric anal sphincter rupture in primiparous women: a comparison between two European delivery settings. Acta Obstet Gynecol Scand 2008;87:209-15

12. Räisänen $S$, Vehviläinen-Julkunen K, Gissler M, et al. Up to sevenfold inter-hospital differences in obstetric anal sphincter injury ratesA birth register-based study in Finland. BMC Res Notes 2010;3:345.

13. Baghestan E, Irgens LM, Børdahl PE, et al. Trends in risk factors for obstetric anal sphincter injuries in Norway. Obstet Gynecol 2010;116:25-34.

14. Andrews $\mathrm{V}$, Shelmeridine $\mathrm{S}$, Sultan $\mathrm{AH}$, et al. Anal and urinary incontinence 4 years after a vaginal delivery. Int Urogynecol J 2013;24:55-60.

15. Faltin DL, Boulvain M, Floris LA, et al. Diagnosis of anal sphincter tears to prevent fecal incontinence: a randomized controlled trial. Obstet Gynecol 2005;106:6-13.

16. Sultan AH, Fernando R. injury Mobstetric. Curr Obstet Gynaecol 2001;11:279-84.

17. Zimmo K, Laine K, Vikanes $\AA$, et al. Diagnosis and repair of perineal injuries: knowledge before and after expert training-a multicentre observational study among Palestinian physicians and midwives. BMJ Open 2017;7:e014183.

18. Fernando RJ, Sultan AH, Freeman RM, et al. The management of third- and fourth- degree perineal tears. Royal College of Obstetricians and Gynaecologists (RCOG) green top guidelines. London, 2015:No. 29.

19. Anderson B, Attwood B, Baird G, et al. Intrapartum care: management and delivery of care to women in labour. National Institute for Health and Clinical Excellence (NICE) Guidelines. London, 2015:No. 55.

20. Hassan S, Vikanes $\mathrm{A}$, Laine $\mathrm{K}$, et al. Building a research registry for studying birth complications and outcomes in six Palestinian governmental hospitals. BMC Pregnancy Childbirth 2017;17:112.

21. Kaplan HC, Brady PW, Dritz MC, et al. The influence of context on quality improvement success in health care: a systematic review of the literature. Milbank Q 2010;88:500-59.

22. Guzmán Rojas RA, Shek KL, Langer SM, et al. Prevalence of anal sphincter injury in primiparous women. Ultrasound Obstet Gynecol 2013;42:n/a-6.

23. Andrews $\mathrm{V}$, Sultan $\mathrm{AH}$, Thakar $\mathrm{R}$, et al. Occult anal sphincter injuriesmyth or reality? BJOG 2006;113:195-200.

24. Fynes M, Donnelly V, Behan M, et al. Effect of second vaginal delivery on anorectal physiology and faecal continence: a prospective study. Lancet 1999;354:983-6.

25. Tucker J, Clifton V, Wilson A. Teetering near the edge; women's experiences of anal incontinence following obstetric anal sphincter injury: an interpretive phenomenological research study. Aust N Z J Obstet Gynaecol 2014;54:377-81.

26. Palestinian Central Bureau of Statistics. Palestinians at the end of 2015. 2015 http://www.pcbs.gov.ps/site/lang_en/881/default. aspx (Retrieved 30 Dec 2015).

27. Sultan $\mathrm{AH}$, Kamm MA, Hudson CN. Obstetric perineal trauma: An audit of training. J Obstet Gynaecol 1995;15:19-23.

28. Hirayama F, Koyanagi A, Mori R, et al. Prevalence and risk factors for third- and fourth-degree perineal lacerations during vaginal delivery: a multi-country study. BJOG 2012;119:340-7.

29. Cabana MD, Rand CS, Powe NR, et al. Why don't physicians follow clinical practice guidelines? A framework for improvement. JAMA 1999;282:1458-65.

30. Fernando RJ, Sultan AH, Radley S, et al. Management of obstetric anal sphincter injury: a systematic review \& national practice survey. BMC Health Serv Res 2002;2:9.

31. Bick DE, Ismail KM, Macdonald S, et al. How good are we at implementing evidence to support the management of birth related perineal trauma? A UK wide survey of midwifery practice. BMC Pregnancy Childbirth 2012;12:57. 